\title{
1 Hip circumduction is not a compensation for reduced knee flexion angle during gait
}

2 Article Type: Original Article

3 Tunc Akbas, PhD, Sunil Prajapati, David Ziemnicki, Poornima Tamma, Sarah Gross, and James

4 Sulzer, PhD

5 Department of Mechanical Engineering, University of Texas at Austin

7 Corresponding Author:

8 Tunc Akbas, PhD

9204 E. Dean Keeton St, Austin, TX 78712

10 tuncakbas@utexas.edu

115128066820

12

13 Keywords: Stroke, Gait, Compensation, Stiff-Knee Gait, Abnormal Coordination, Neural control 14 of movement

15

16 Word count (Introduction through Discussion): 3,727 
17

\section{Abstract}

It has long been held that hip abduction compensates for reduced swing-phase knee flexion angle, especially in those after stroke. However, there are other compensatory motions such as pelvic obliquity (hip hiking) that could also be used to facilitate foot clearance with greater energy efficiency. Our previous work suggested that hip abduction may not be a compensation for reduced knee flexion after stroke. Previous study applied robotic knee flexion assistance in people with post-stroke Stiff-Knee Gait (SKG) during pre-swing, finding increased abduction despite improved knee flexion and toe clearance. Thus, our hypothesis was that hip abduction is not a compensation for reduced knee flexion. We simulated the kinematics of post-stroke SKG on unimpaired individuals with three factors: a knee orthosis to reduce knee flexion, an ankle-foot orthosis commonly worn by those post-stroke, and matching gait speeds. We compared spatiotemporal measures and kinematics between experimental factors within healthy controls and with a previously recorded cohort of people with post-stroke SKG. We focused on frontal plane motions of hip and pelvis as possible compensatory mechanisms. We observed that regardless of gait speed, knee flexion restriction significantly increased pelvic obliquity $\left(2.79^{\circ}, \mathrm{p}<0.01\right)$ compared to unrestricted walking $\left(1.5^{\circ}, \mathrm{p}<0.01\right)$, but similar to poststroke SKG $\left(3.4^{\circ}\right)$. However, those with post-stroke SKG had significantly greater hip abduction $\left(8.2^{\circ}\right)$ compared to unimpaired individuals with restricted knee flexion $\left(4.2^{\circ}, p<0.05\right)$. These results show that pelvic obliquity, not hip abduction, compensates for reduced knee flexion angle. Thus, other factors, possibly neural, facilitate exaggerated hip abduction observed in post-stroke SKG. 


\section{Introduction}

Stroke causes numerous impairments, including muscle weakness, spasticity, abnormal muscle coordination and altered proprioception, resulting in walking disorders. Stiff-knee gait

41 (SKG), defined as reduced peak knee flexion angle during swing phase of the paretic side, is a

42 common walking disorder following stroke (Deirdre Casey Kerrigan, Gronley, \& Perry, 1991).

43 Gait researchers have long assumed that people with post-stroke SKG compensate for reduced

44 knee flexion with increased hip hiking, hip circumduction and/or vaulting (D Casey Kerrigan,

45 Frates, Rogan, \& Riley, 2000; Deirdre Casey Kerrigan et al., 1991; Perry \& Burnfield, 1992).

However, the causal relations between these compensations and reduced foot clearance have never been established. Stroke patients exhibit similar or higher foot clearance values compared to healthy individuals (Little, McGuirk, \& Patten, 2014; Matsuda et al., 2017) suggesting one or more of these aforementioned compensations could be redundant. It is a commonly held belief that hip abduction, the main frontal plane component of hip

51 circumduction, compensates for lack of knee flexion (Perry \& Burnfield, 1992). However, the

52 substantial energetic cost of hip abduction (Shorter, Wu, \& Kuo, 2017) could make it the least

53 desirable compensatory motion. Thus, in this work we investigated the necessity of hip abduction as a compensatory motion for reduced knee flexion.

Our previous work suggests excessive hip abduction may not be a compensation, but possibly the result of an abnormal coordination pattern emerging after stroke. We used a knee

57 flexion torque assistance during pre-swing and observed an increased hip abduction angle 
foot clearance suggested that abduction was not acting as a gait compensation. Other possible causes such as loss of balance, spasticity and reduced proprioception could not account for this phenomenon. Rather, excessive hip abduction could be part of a cross-planar abnormal coordination pattern, for example, reflex-based (Finley, Perreault, \& Dhaher, 2008) or voluntary synergies (Cruz \& Dhaher, 2008; Cruz, Lewek, \& Dhaher, 2009; Neckel, Blonien, Nichols, \& Hidler, 2008). Descriptive analyses of post-stroke gait have associated abnormal coordination with gait dysfunction. For instance, Clark et al. used non-negative matrix factorization to show that the number of coordination patterns in post-stroke negatively correlates with locomotor performance and clinical assessments compared to healthy individuals (Clark, Ting, Zajac, Neptune, \& Kautz, 2010). Cases with fewer modules observed abductor activity coupled with sagittal plane muscles. Thus, accumulating evidence points to post-stroke hip abduction as part of abnormal coordination.

The concept of hip abduction in post-stroke SKG as an abnormal coordination pattern would be at odds with the widely accepted hypothesis that hip abduction is a compensation for reduced knee flexion (Perry \& Burnfield, 1992). Here, we challenge the latter claim by determining how healthy individuals react to kinematically constrained knee flexion. If, as our earlier data suggest, abduction is not a compensatory motion, then we would expect healthy

77 individuals to adapt to reduced knee flexion using other compensations, such as increased hip hiking (pelvic obliquity) or vaulting (increased plantarflexion of contralateral ankle during swing). healthy individuals with an adjustable knee brace and observed the resulting compensations. 
82 Mechanical induction of gait deviations has been used to successfully quantify gait asymmetry

83 (Shorter, Polk, Rosengren, \& Hsiao-Wecksler, 2008) and evaluate energy expenditure (Hanada

84 \& Kerrigan, 2001). Lewek et al. mechanically induced SKG using a knee brace, finding that such

85 a constraint results in higher metabolic cost (Lewek, Osborn, \& Wutzke, 2012). Here we took a

86 similar approach, but instead we examined how traditional compensatory parameters vary

87 between those with post-stroke SKG and those with mechanically induced SKG. We additionally

88 introduced other factors to more accurately simulate post-stroke gait, for example we matched

89 walking speeds and added an ankle-foot orthosis commonly worn by individuals post-stroke.

90 We predicted that more energy efficient compensations to reduce knee flexion, i.e. hip hiking,

91 would facilitate foot clearance instead of abduction in healthy individuals. Differing reactions

92 between post-stroke and restricted healthy individuals to similar knee motion would suggest

93 that hip abduction is not a compensation for reduced knee flexion. This work distinguishes the

94 impairment-related and compensatory joint motions in post-stroke gait, which will lead to

95 improved clinical assessments and targeted therapy.

\section{Methods}

98 Twelve unimpaired healthy individuals with no prior musculoskeletal injury gave written

99 informed consent according to the guidelines approved by the University of Texas at Austin

100 Institutional Review Board to participate in the experiment (Table S1). 
103 SKG in previous study, where all participants were left-sided hemiparetics with knee range of 104 motion at least $16^{\circ}$ less on the effected side during swing phase (Sulzer et al., 2010). Since all 105 patients had reduced knee flexion angle during swing, we restricted the knee with a 106 commercial knee brace (Comfortland Medical, Mebane, NC) with a range-of-motion setting 107 nominally at $0^{\circ}$. Since half of our patient sample wore an ankle-foot orthosis (AFO), we incorporated a commonly used AFO (Ossur, Reykjavík, Iceland) setting the ankle in a neutral, $90^{\circ}$ ankle flexion position. Both orthoses were implemented on the left side to match the patient sample. We also further imitated our sample by matching gait speeds at $0.5 \mathrm{~m} / \mathrm{s}$. Thus, we used a $2 \times 2 \times 2$ factorial design consisting of the factors of knee restriction, ankle restriction

112 and walking speed. All subjects walked on a split-belt force treadmill (Bertec, Columbus, $\mathrm{OH}$ ),

113 which recorded ground reaction forces. Lower limb kinematic data were collected using an 114 optical motion capture system (PhaseSpace Motion Capture, San Leandro, CA). Each of the 115 three experimental factors consisted of two levels resulting in eight total conditions. Each 116 healthy participant walked for three minutes for each condition, approximately 150 steps for

117 slow walking speed and 200 steps for normal walking speed without receiving any prior 118 practice. The Restricted condition consisted of both knee restriction and ankle restriction. The 119 knee brace restriction was also implemented without the use of an AFO (Brace), and conversely 120 the AFO was implemented without knee brace restriction (AFO). Lastly, subjects walked with no 121 restriction at all while wearing the brace (Free). Each condition was implemented with slow (0.5 $122 \mathrm{~m} / \mathrm{s})$ and normal $(1 \mathrm{~m} / \mathrm{s})$ walking speeds, representing the walking speed of the post-stroke SKG 123 cohort and typical comfortable healthy walking speed, respectively. The order of the conditions 124 was randomized. Motion capture data was collected at $240 \mathrm{~Hz}$ and force measures from the 
125 instrumented treadmill were collected at $1 \mathrm{kHz}$. Figure 1 shows the representative overview of

126 experimental setup demonstrating the Restricted condition. The data of nine individuals with

127 post-stroke SKG collected from the baseline stage of previous study (Sulzer et al., 2010) was

128 used to represent post-stroke SKG where participants walked at $0.5 \mathrm{~m} / \mathrm{s}$ for two minutes.

Kinematic and Spatiotemporal Measures

Data was separated into gait cycles using left heel strikes for each participant corresponding to the given condition-speed pair. The heel-strike was detected using the instrumented split-belt treadmill based on a vertical force threshold of $10 \mathrm{~N}$. The first 20 gait cycles were discarded to account for adaptation to the condition. Knee flexion, ankle plantarflexion and hip abduction

134 angles of the ipsilateral (constrained/paretic) and contralateral (unconstrained/non-paretic)

135 sides along with pelvic obliquity was extracted from a random selection of 25 gait cycles of each

136 healthy participant for each condition to match the number of gait cycles collected from the

137 individuals with post-stroke SKG from the previous experiment. We quantified hip

138 circumduction as the hip abduction angle as opposed to the lateral displacement of malleolus

139 (Lehmann, Condon, \& Price, 1987) and coronal thigh angle (D Casey Kerrigan et al., 2000). The

140 hip hiking is quantified by the coronal angle of the pelvis defined as pelvic obliquity (Michaud,

141 Gard, \& Childress, 2000). Range-of-motion (ROM) for each movement was defined as the

142 difference between minimum and maximum joint angle measures in positive directions during

143 pre-swing and swing phases of the gait cycle. The contralateral plantarflexion angle at toe-off

144 was extracted to measure the amount of vaulting. Spatiotemporal characteristics were

145 obtained including maximum toe height, maximum toe width, and toe height and width at

146 minimum toe clearance from the ipsilateral (constrained/paretic) side (Figure 2). The maximum 
147 toe height was defined as the maximum vertical displacement of the toe marker. Maximum toe

148 width was defined as the maximum lateral displacement of toe marker. Minimum toe clearance

149 was quantified as the local minimum vertical displacement during swing phase (Winter, 1991).

150 Finally, toe width at minimum toe clearance was quantified as the lateral displacement of toe

151 marker at minimum toe clearance. Step asymmetry was quantified by the ratios of pre-swing

152 times and swing times between ipsilateral (constrained/paretic) and contralateral

153 (unconstrained/non-paretic) sides (Figure 2). Swing and pre-swing ratios were calculated by the

154 ratio between swing phases of opposite limbs and the ratio between the durations in double

155 support periods prior to swing of the corresponding limb respectively.

157 Statistical Analysis

158 The collected data was analyzed using a linear mixed model (Ime4) package (R Development

159 Core Team, 2008). The first model included only the healthy participant pool. This model

160 included the ROM measures from knee flexion, hip abduction and ankle plantarflexion of

161 ipsilateral (constrained/paretic) side, and pelvic obliquity and spatiotemporal measures as

162 dependent variables, with fixed effects of knee restriction, ankle restriction and walking speed.

163 A linear mixed-effects model using the aforementioned factors, participant as a random effect

164 and followed by Tukey-Kramer post hoc testing to evaluate the significance of the differences in

165 the outcome variables between factors $(\alpha<0.05)$.

166 The second model implemented the same linear mixed-effects model with the healthy

167 participant pool at slow walking speed $(0.5 \mathrm{~m} / \mathrm{s})$ with Free and Brace conditions as well as

168 people with post-stroke SKG. We have accounted for repeated measures between Free and 
Brace conditions within the healthy group in this model using the same labels for the participants. Similar to the analysis in healthy individuals, we conducted Tukey-Kramer post hoc testing with the joint angle ROM measures and spatiotemporal measures as dependent variables. We examined differences between the participants with post-stroke SKG from the previously collected data and the two healthy conditions at matched speeds (Free and Brace). We ran the Shapiro-Wilk normality test for all the outcome measures within corresponding factors for the first model and corresponding groups for the second model to confirm the normality of the data sets $(p>0.05)$.

\section{Results}

\section{Comparisons within healthy individuals}

Average gait trajectories for all the aforementioned kinematic measures for all subjects in Free, Brace, AFO and Restricted conditions with slow and normal walking speeds are shown in Figure 3. A summary of the outcome measures for each condition can be found in Table S2. The following highlights the statistical comparisons based on the linear-mixed model.

We observed a main effect of knee restriction on knee flexion $\operatorname{ROM}\left(F_{(1,76)}=158, p<.001\right)$ and ankle flexion $\operatorname{ROM}\left(F_{(1,76)}=16.4, p<.001\right)$, both reduced significantly in Brace condition $(t=$ -12.6, $p<.001, t=4.35, p<.001$, respectively) compared to Free condition. Knee restriction also affected pelvic obliquity $\left(F_{(1,76)}=17.1, p<.001\right)$ but there was no significant difference in hip abduction $\left(F_{(1,76)}=2.27, p=.136\right)$. For instance, pelvic obliquity ROM increased in Brace condition compared to the Free condition $(t=5.26, p<.001)$. We observed the main effect of 
ankle restriction on ankle plantarflexion $\operatorname{ROM}\left(F_{(1,76)}=6.00, p=.017\right)$, significantly reduced from the Free condition to the AFO condition ( $t=-2.61, p=.006)$. As expected, ankle restriction did not affect compensatory parameters such as hip abduction $\left(F_{(1,76)}=0.01, p=.908\right)$ or pelvic obliquity $\left(F_{(1,76)}=0.41, p=.53\right)$. Vaulting was not affected by knee restriction $\left(F_{(1,76)}=0.05, p=\right.$ $.824)$ or ankle restriction $\left(F_{(1,76)}=0.14, p=.708\right)$. When comparing the joint angle ROM measures between the Brace and Restricted conditions, i.e. the interactive effect of the ankle and knee restriction, there was no significant interaction effect for any of the measures ( $p$ > $.05)$.

As expected, walking speed affected multiple kinematic variables, including knee flexion $\operatorname{ROM}\left(F_{(1,76)}=9.46, p=.003\right)$ and ankle flexion $\left(F_{(1,76)}=5.07, p=.042\right)$, both decreased significantly in slow walking speed $(t=-3.07, p=.003, t=--2.06, p<.042$, respectively). However, we did not observe any significant interactive effects between the walking speed and restrictions on knee and ankle $(p>.05)$. That is, the change in speed did not modulate the relations between kinematic variables reported above.

Maximum step height was significantly affected by knee restriction $\left(F_{(1,76)}=15.94, p<.001\right)$ and toe height at minimum toe clearance (HMTC) was significantly affected by ankle restriction $\left(F_{(1,76)}=6.91, p<.010\right)$. The maximum step height was significantly decreased in Brace condition $(t=-3.84, p<.001)$ compared to the Free condition, whereas HMTC was significantly increased in AFO $(t=2.41, p<.018)$ condition compared to Free condition. On the other hand, the maximum step width was significantly affected by knee restriction $\left(F_{(1,76)}=6.25, p=.015\right)$ whereas no significant effect was observed in ankle restriction $\left(F_{(1,76)}=0.01, p=.918\right)$. The maximum step width was increased in the Brace condition ( $t=2.45, p=.017)$ compared to the 
212 Free condition. In terms of gait symmetry, the swing-time ratio was only affected by knee

213 restriction $\left(F_{(1,76)}=14.94, p<.001\right)$ and pre-swing time ratio was only affected by ankle

214 restriction $\left(F_{(1,1959)}=5.68, p=.012\right)$. The swing time ratio was significantly increased in the Brace

215 condition $(t=3.86, p<.001)$ and the pre-swing time ratio was significantly decreased in the

216 AFO condition ( $t=-2.38, p=.020)$ compared to the Free condition.

As expected, walking speed affected multiple spatiotemporal measures, including maximum

step height $\left(F_{(1,76)}=14.5, p<.001\right)$ and $\operatorname{HMTC}\left(F_{(1,76)}=10.1, p=.002\right)$, both decreasing

219 significantly in slow walking speed $(t=-3.78, p=.003, t=-3.22, p<.002$, respectively). We did

220 not observe any significant interactive effects between the walking speed and restrictions on

221 knee and ankle $(p>.05)$ for spatiotemporal measures, similar to the interactive effects between

222 walking speed and knee and ankle joint ROM measures.

Comparisons to post-stroke SKG

We compared the kinematic and spatiotemporal outcomes between the Brace and Free

227 speed. Our analysis only focused on the Brace condition because it is a more well-controlled

228 condition compared to both ankle and knee restriction. Furthermore, post-hoc analysis did not

229 indicate any significant differences between the Brace and Restricted conditions for kinematic

230 and spatiotemporal outcome measures. Gait trajectories are illustrated in Figure 4 and

231 corresponding ROM measures were shown in Supplementary Figure 1 and statistical 
comparisons between groups are shown in Table 1. The following is a summary of the statistical comparisons in selected parameters.

The knee flexion ROM in the Brace condition and $S K G$ were not significantly different $(t=$ $0.73, p=.467)$ but the $S K G$ group was significantly lower than the Free condition $(t=8.88, p=$ .001). Similarly, there were no significant differences in ankle plantarflexion ROM between the Brace condition and $S K G(t=0.26, p=.788)$. However, the Free condition had significantly higher ankle plantarflexion compared to the SKG group $(t=2.78, p<.013)$. There was no significant difference in pelvic obliquity ROM between the Brace condition and SKG group $(t=3.02, p=.103)$ but the $S K G$ group was significantly higher than the Free condition $(t=5.25, p$ $<$.001). In addition, hip abduction in post-stroke SKG was significantly higher than the Brace condition $(t=2.52, p=.012)$. There was no significant difference in vaulting between the SKG group and Brace condition ( $t=0.53, p=.585$ ) or between the $S K G$ group and Free condition ( $t=$ $0.93, p=.351)$. Spatiotemporal characteristics differed between groups. The maximum toe height was significantly higher for the Free condition compared to the SKG $(t=4.99, p<.001)$. Maximum toe width in the Free condition was significantly lower than the $S K G$ group $(t=3.13, p<.001)$. The toe height at minimum toe clearance in the SKG group was significantly higher than both the Free $(t=4.08, p<.001)$ and Brace conditions $(t=3.49, p<.001)$. Toe width measures at minimum toe clearance in $S K G$ was significantly higher compared to the Free $(t=3.79, p<.001)$ and Brace $(t=4.38, p<.001)$ conditions. 
The pre-swing time ratio in SKG group was significantly higher compared to Free ( $t=5.74, p$

$253<.001)$ and Brace conditions $(t=5.34, p<.001)$. Similarly, swing ratio in SKG group was

254 significantly higher than the Brace condition $(t=7.57, p<.001)$ and the Free condition $(t=3.92$,

$255 p<.001)$.

\section{Discussion}

Hip abduction has long been assumed to be a compensation for reduced knee flexion angle during swing, and accordingly, excessive abduction in post-stroke SKG has been attributed to the same mechanism (Perry \& Burnfield, 1992). To test this hypothesis, we applied kinematic constraints at the knee and ankle in healthy gait to imitate the sagittal plane kinematics of post-

261 stroke SKG and evaluated resulting compensations. In response to reduced knee flexion,

262 healthy individuals compensated with greater pelvic obliquity with no significant change in hip

263 abduction, regardless of gait speed. Individuals with post-stroke SKG exhibited a similar

264 increase in pelvic obliquity, but in contrast to healthy individuals with constrained knee flexion,

265 higher hip abduction was observed. In addition, the minimum toe clearance of people with

266 post-stroke SKG was also higher than those with mechanically induced SKG, indicating that the

267 excessive abduction was not necessary for foot clearance. In summary, our results show that

268 hip abduction is not a gait compensation for reduced knee flexion angle.

271 motion. Stroke participants exhibited the same sagittal plane joint ROM as healthy individuals

272 with restricted knee motion and also exhibited the same compensatory motions indicated by 
273 the pelvic obliquity ROM. However, in stark contrast to healthy individuals with mechanically

274 reduced knee flexion, those with post-stroke SKG walked with substantially higher hip

275 abduction and toe clearance, seemingly with no biomechanical benefit. Previous work has

276 reported excessive contributions of the ankle plantarflexor (soleus) and abductor muscles

277 (gluteus medius) during forward propulsion and swing phase of the paretic side during post-

278 stroke gait, indicating abnormal coordination similar to what we hypothesized (Hall, Peterson,

279 Kautz, \& Neptune, 2011). Our own work revealed that increased knee flexion angle and toe

280 clearance provided by exoskeletal assistance resulted in greater hip abduction in post-stroke

281 SKG which could not be accounted by biomechanical factors (Sulzer et al., 2010). Further

282 analysis suggested that a cross-planar reflex coupling initiated by spastic rectus femoris co-

283 activated with gluteus medius (Akbas, Neptune, \& Sulzer, In review). Thus while excessive hip

284 abduction should be expected to avoid excessive ankle plantarflexion, for example, during

285 equinus deformity of the foot (Kinsella \& Moran, 2008), the cause of excessive abduction in

286 those with only reduced knee flexion could be due to non-biomechanical causes such as

287 abnormal coordination (Brunnström, 1970).

Instead of abduction, we found increased pelvic obliquity as the primary compensation for reduced knee flexion. Earlier work has shown only increased pelvic obliquity during toe-off in

290 post-stroke gait compared to healthy gait, without any significant changes in hip abduction

291 (Cruz et al., 2009; Matsuda et al., 2016). Further, in those with post-stroke hemiparesis, pelvic

292 elevation and knee flexion were inversely correlated with the walking speed, whereas no

293 correlation was observed with hip abduction (Stanhope, Knarr, Reisman, \& Higginson, 2014).

294 Not only does this evidence suggest that pelvic obliquity is the primary compensation for 
295 reduced foot clearance, but additional work indicates the cost of abduction (Shorter et al.,

296 2017). Increased circumduction magnitude is exponentially correlated with the cost of

297 metabolic energy during walking (Shorter et al., 2017). Our results in healthy individuals

298 conclusively add to this literature by directly illustrating that pelvic obliquity is the primary

299 compensation for reduced knee flexion, whereas hip abduction is not a compensation.

There were additional differences between post-stroke and healthy individuals. For gait with restricted knee motion. This difference can be explained by the increased swing time due to excessive abduction. Increased minimum toe clearance in post-stroke SKG compared to those with mechanically restricted knee motion indicates an overcompensation following toeclearance. This overcompensation could be due to the lack of proprioceptive feedback

306 following post-stroke hemiparesis (Keenan, Perry, \& Jordan, 1983). Alternatively, the increased 307 toe height at minimum toe clearance could be due to ankle impairments (Basmajian, Kukulka, 308 Narayan, \& Takebe, 1975; Olney, Griffin, Monga, \& McBride, 1991). 
316 could contribute to increased hip abduction in post-stroke SKG, it is unlikely this would result in

317 such an energetically costly compensation over a long period of time.

318 In conclusion, our data shows that hip abduction is not a necessary compensation for

319 reduced knee flexion during gait, in direct opposition to widely held beliefs of abduction's

320 compensatory role. Instead, pelvic obliquity is the primary compensatory motion associated

321 with reduced swing phase knee flexion ROM. Together with previous findings, these data

322 suggest that excessive hip abduction in those with post-stroke SKG could originate from a non-

323 biomechanical cause, such as an abnormal coordination pattern. The correct characterization of

324 compensation and impairment will lead towards improved treatment strategies and

325 interventions. 
Akbas, T., Neptune, R. R., \& Sulzer, J. (In review). Neuromusculoskeletal simulation reveals abnormal rectus femoris-gluteus medius reflex coupling in post-stroke gait. Stroke.

Basmajian, J., Kukulka, C., Narayan, M., \& Takebe, K. (1975). Biofeedback treatment of foot-drop after stroke compared with standard rehabilitation technique: effects on voluntary control and strength. Archives of Physical Medicine and Rehabilitation, 56(6), 231-236.

Brunnström, S. (1970). Movement therapy in hemiplegia: a neurophysiological approach: Facts and Comparisons.

Clark, D. J., Ting, L. H., Zajac, F. E., Neptune, R. R., \& Kautz, S. A. (2010). Merging of healthy motor modules predicts reduced locomotor performance and muscle coordination complexity poststroke. Journal of neurophysiology, 103(2), 844-857.

Cruz, T. H., \& Dhaher, Y. Y. (2008). Evidence of Abnormal Lower-Limb Torque Coupling After Stroke An Isometric Study. Stroke, 39(1), 139-147.

Cruz, T. H., Lewek, M. D., \& Dhaher, Y. Y. (2009). Biomechanical impairments and gait adaptations poststroke: multi-factorial associations. Journal of biomechanics, 42(11), 1673-1677.

Finley, J. M., Perreault, E. J., \& Dhaher, Y. Y. (2008). Stretch reflex coupling between the hip and knee: implications for impaired gait following stroke. Experimental brain research, 188(4), 529-540.

Hall, A. L., Peterson, C. L., Kautz, S. A., \& Neptune, R. R. (2011). Relationships between muscle contributions to walking subtasks and functional walking status in persons with post-stroke hemiparesis. Clinical biomechanics, 26(5), 509-515.

Hanada, E., \& Kerrigan, D. C. (2001). Energy consumption during level walking with arm and knee immobilized. Archives of physical medicine and rehabilitation, 82(9), 1251-1254.

Keenan, M. A., Perry, J., \& Jordan, C. (1983). Factors affecting balance and ambulation following stroke. Clinical Orthopaedics and Related Research(182), 165-171.

Kerrigan, D. C., Frates, E. P., Rogan, S., \& Riley, P. O. (2000). Hip hiking and circumduction: quantitative definitions. American journal of physical medicine \& rehabilitation, 79(3), 247-252.

Kerrigan, D. C., Gronley, J., \& Perry, J. (1991). Stiff-legged gait in spastic paresis. A study of quadriceps and hamstrings muscle activity. American journal of physical medicine \& rehabilitation, 70(6), 294-300.

Kinsella, S., \& Moran, K. (2008). Gait pattern categorization of stroke participants with equinus deformity of the foot. Gait \& posture, 27(1), 144-151.

Lehmann, J. F., Condon, S. M., \& Price, R. (1987). Gait abnormalities in hemiplegia: their correction by ankle-foot orthoses. Archives of Physical Medicine and Rehabilitation, 68(11), 763-771.

Lewek, M. D., Osborn, A. J., \& Wutzke, C. J. (2012). The influence of mechanically and physiologically imposed stiff-knee gait patterns on the energy cost of walking. Archives of physical medicine and rehabilitation, 93(1), 123-128.

Little, V. L., McGuirk, T. E., \& Patten, C. (2014). Impaired limb shortening following stroke: what's in a name? PloS one, 9(10), e110140.

Matsuda, F., Mukaino, M., Ohtsuka, K., Tanikawa, H., Tsuchiyama, K., Teranishi, T., . . Saitoh, E. (2016). Analysis of strategies used by hemiplegic stroke patients to achieve toe clearance. Japanese Journal of Comprehensive Rehabilitation Science, 7, 111-118.

Matsuda, F., Mukaino, M., Ohtsuka, K., Tanikawa, H., Tsuchiyama, K., Teranishi, T., . . Saitoh, E. (2017). Biomechanical factors behind toe clearance during the swing phase in hemiparetic patients. Topics in stroke rehabilitation, 24(3), 177-182. 
Michaud, S. B., Gard, S. A., \& Childress, D. S. (2000). A preliminary investigation of pelvic obliquity patterns during gait in persons with transtibial and transfemoral amputation. Journal of rehabilitation research and development, 37(1), 1-10.

Neckel, N. D., Blonien, N., Nichols, D., \& Hidler, J. (2008). Abnormal joint torque patterns exhibited by chronic stroke subjects while walking with a prescribed physiological gait pattern. Journal of neuroengineering and rehabilitation, 5(1), 19.

Olney, S. J., Griffin, M. P., Monga, T. N., \& McBride, I. D. (1991). Work and power in gait of stroke patients. Archives of physical medicine and rehabilitation, 72(5), 309-314.

Perry, J., \& Burnfield, J. (1992). Gait analysis: normal and pathological function. 1992. Thorofare, NJ: Slack.

Shorter, K. A., Polk, J. D., Rosengren, K. S., \& Hsiao-Wecksler, E. T. (2008). A new approach to detecting asymmetries in gait. Clinical Biomechanics, 23(4), 459-467.

Shorter, K. A., Wu, A., \& Kuo, A. D. (2017). The high cost of swing leg circumduction during human walking. Gait \& Posture, 54, 265-270.

Stanhope, V. A., Knarr, B. A., Reisman, D. S., \& Higginson, J. S. (2014). Frontal plane compensatory strategies associated with self-selected walking speed in individuals post-stroke. Clinical Biomechanics, 29(5), 518-522.

Sulzer, J. S., Gordon, K. E., Dhaher, Y. Y., Peshkin, M. A., \& Patton, J. L. (2010). Preswing knee flexion assistance is coupled with hip abduction in people with stiff-knee gait after stroke. Stroke, 41(8), 1709-1714.

Winter, D. A. (1991). Biomechanics and motor control of human gait: normal, elderly and pathological. 


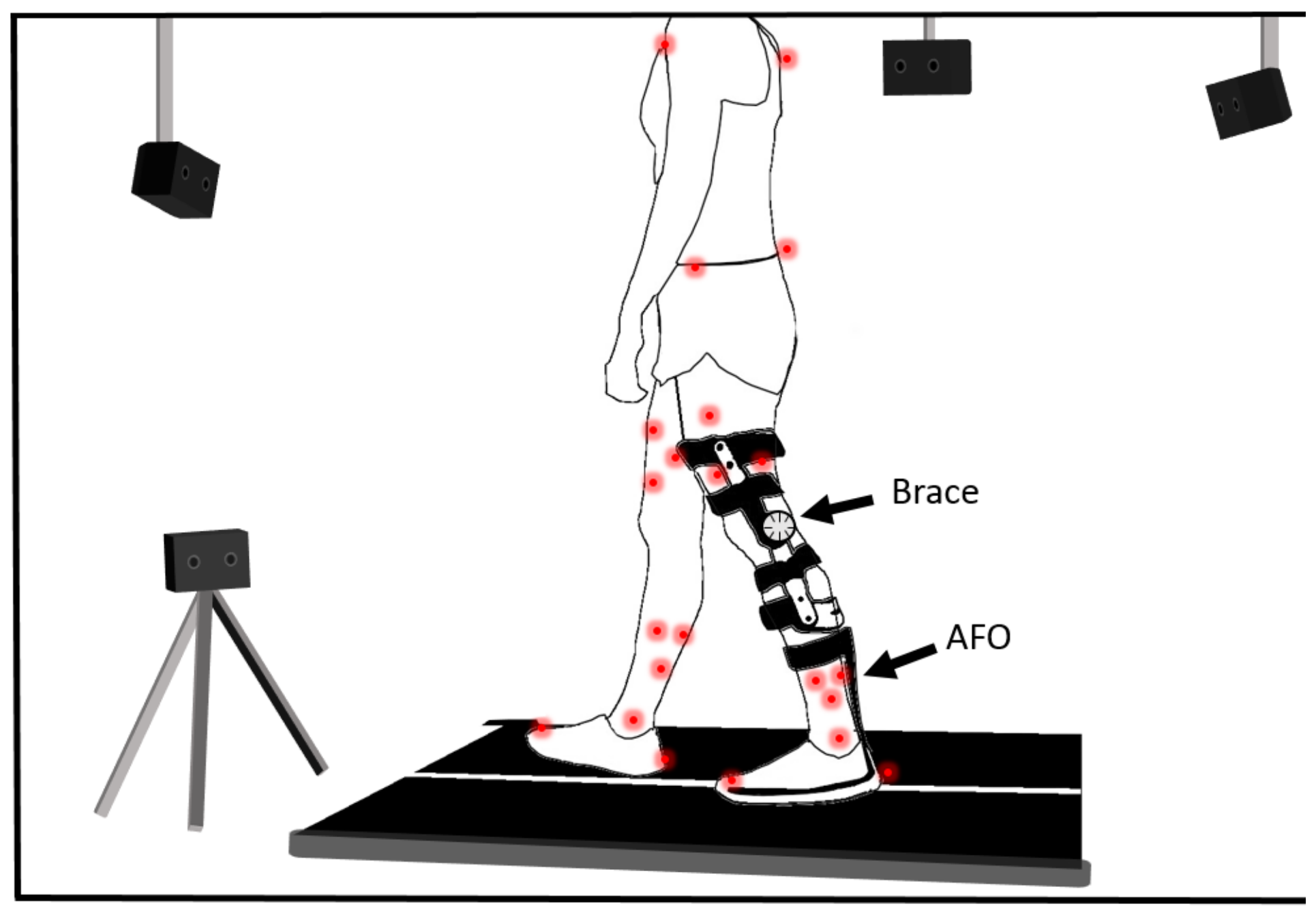

Figure 1: Schematic of experimental setup of mechanically induced SKG. A commercial medical

knee brace and ankle foot orthosis (AFO) are used to imitate reduced knee flexion and orthotic assistance in post-stroke SKG, respectively. For the Free condition, the AFO and flexion angle constraint on the knee brace were removed, whereas for the Brace condition only the AFO was removed, and for the AFO condition only the flexion angle constraint was removed. Position of individual body segments was recorded with active LED markers (shown in red circles) via infrared cameras and gait events were detected using the force measures from the instrumented split-belt treadmill. 


\section{Gait Cycle}

Transverse plane
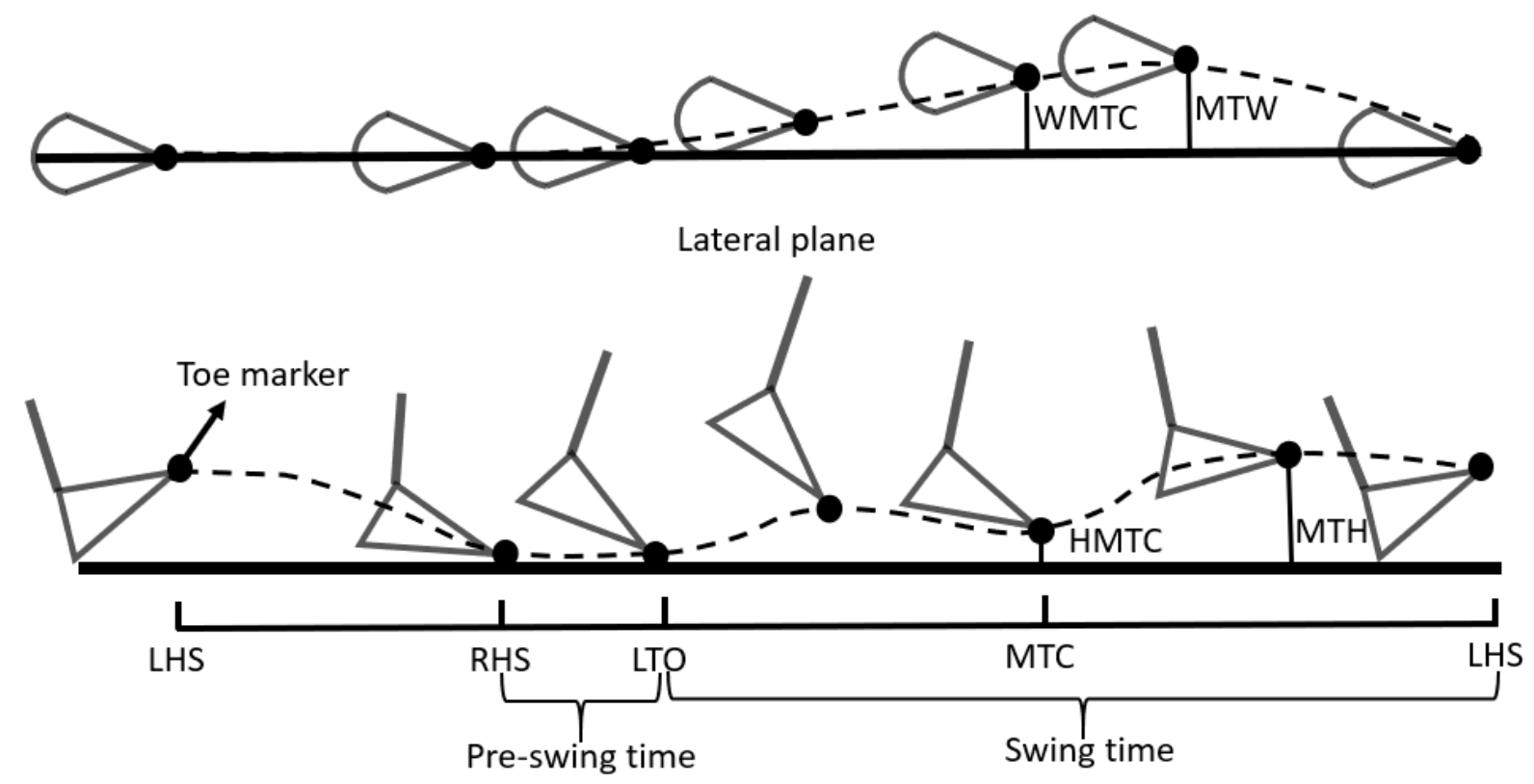

Figure 2: Selected spatiotemporal gait measures illustrated by left toe marker trajectory from

lateral and transverse plane views. Maximum toe height (MTH), maximum toe width (MTW)

and toe height (HMTC) and width (WMTC) measures at minimum toe clearance were obtained

using the toe marker at the corresponding time instances during swing of the

constrained/paretic side. The ratio of swing times (SR) and pre-swing times (PR) between

constrained/paretic and unconstrained/non-paretic sides were used to measure gait

asymmetry. 


\section{Slow}
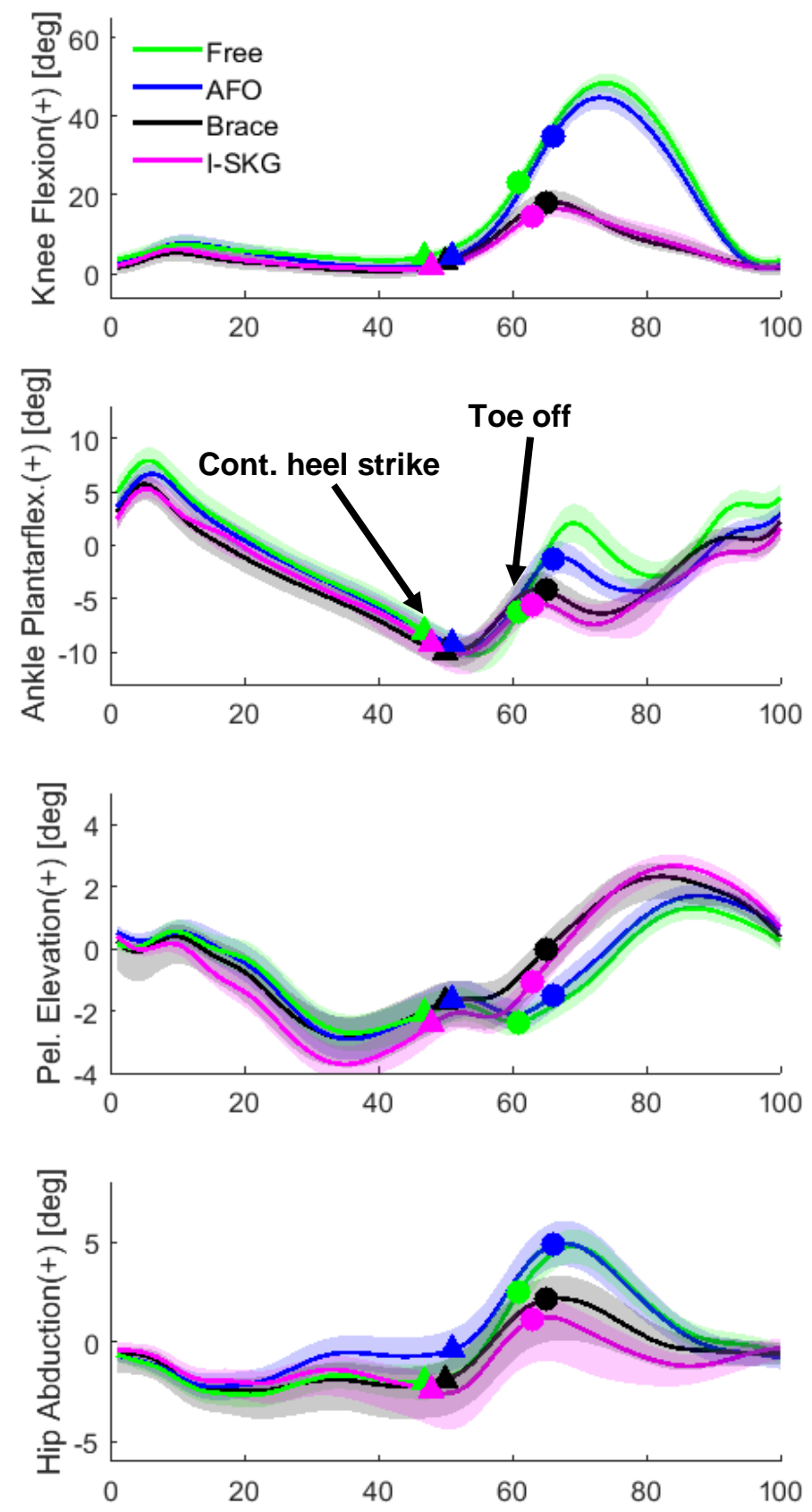

Gait cycle [\%]
Normal
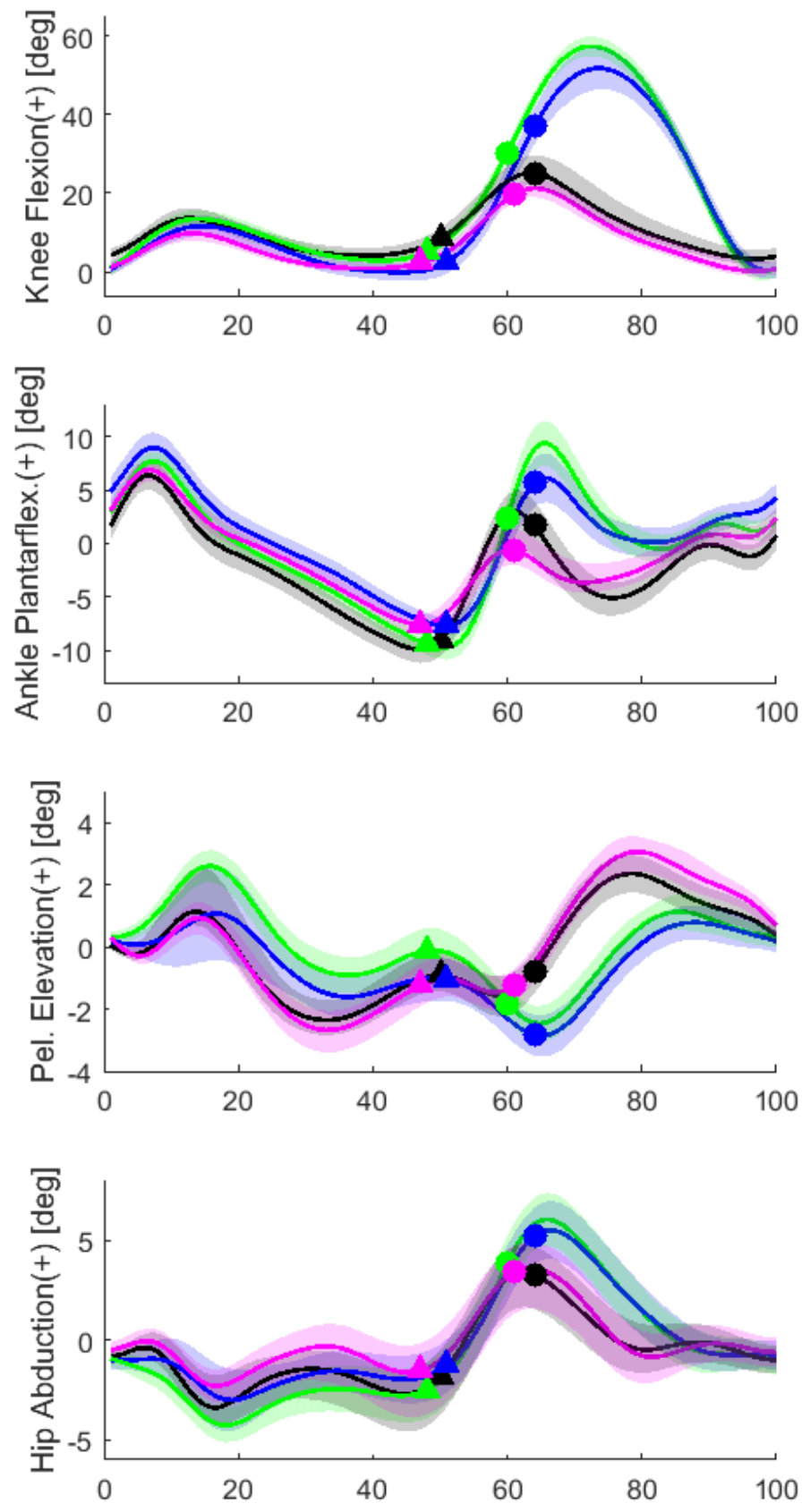

Gait cycle [\%]

Figure 3: Healthy individuals compensate with increased pelvic obliquity and decreased ankle plantarflexion in response to reduced knee flexion. The knee flexion, ankle plantarflexion, pelvic obliquity and hip abduction angles for the constrained side under different conditions (Free, AFO, Brace and Restricted) are given for slow (left) and normal (right) walking speeds. The mean values and the standard errors are shown by solid lines and shaded areas, respectively. 
Contralateral heel strike is delineated by triangles and toe-off by circles. Regardless of walking speed, the introduction of knee restriction increased pelvic obliquity and decreased hip abduction in healthy controls. 
Figure 4
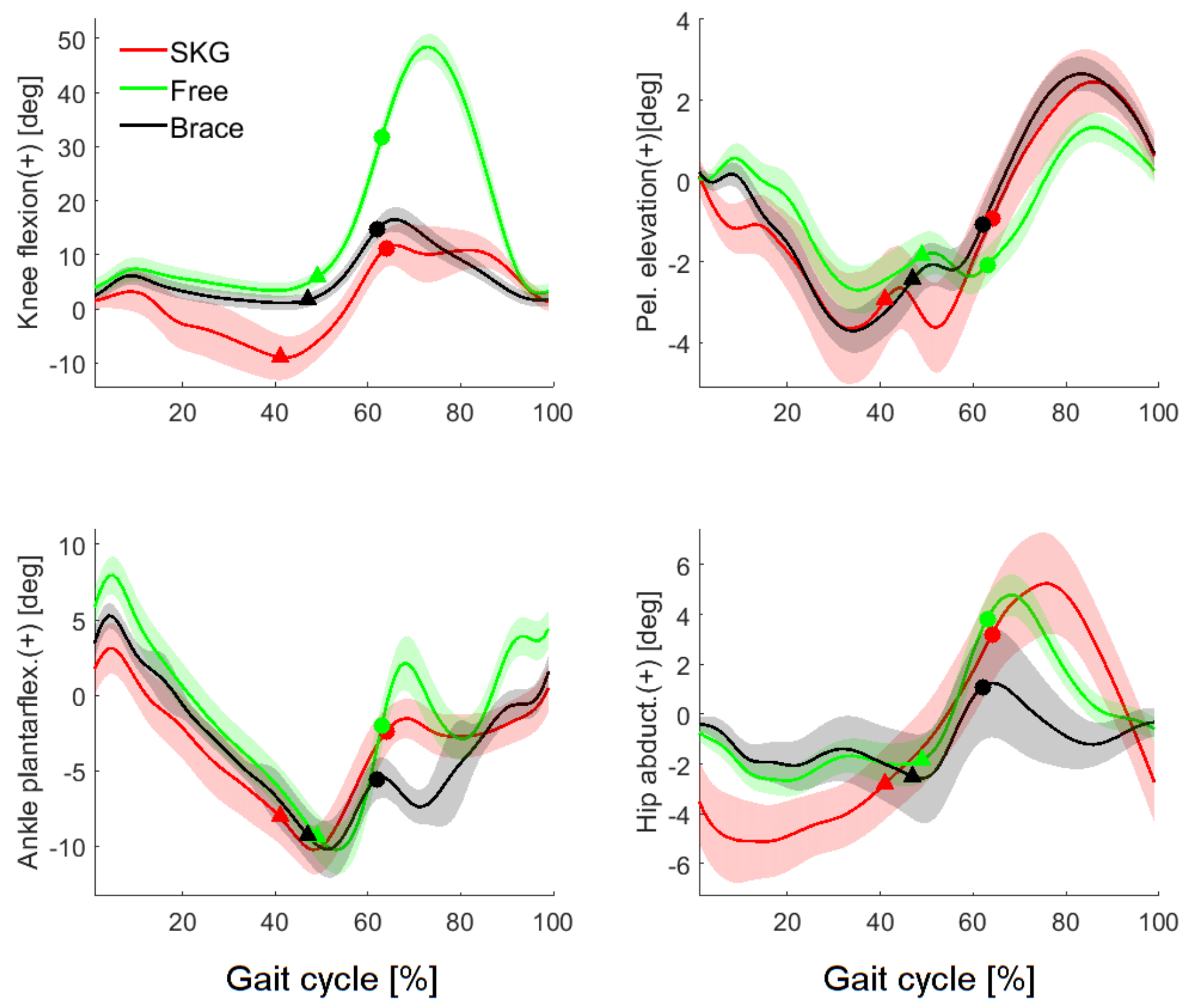

Figure 4: Increased hip abduction angle in stroke contrasts with decreased abduction in the

Brace condition. The knee flexion, hip abduction and ankle flexion angles for the constrained/paretic side and pelvic obliquity for healthy individuals with Free and Brace conditions speed-matched with people with post-stroke SKG. The standard errors are shown by shaded areas. Contralateral heel strike is delineated by triangles and toe-off by circles. Note that 
bioRxiv preprint doi: https://doi.org/10.1101/520684; this version posted January 15,2019 . The copyright holder for this preprint (which was not certified by peer review) is the author/funder. All rights reserved. No reuse allowed without permission.

despite similar knee flexion between the Brace condition and SKG group, the Brace condition resulted in reduced hip abduction while the $S K G$ group had increased hip abduction. 


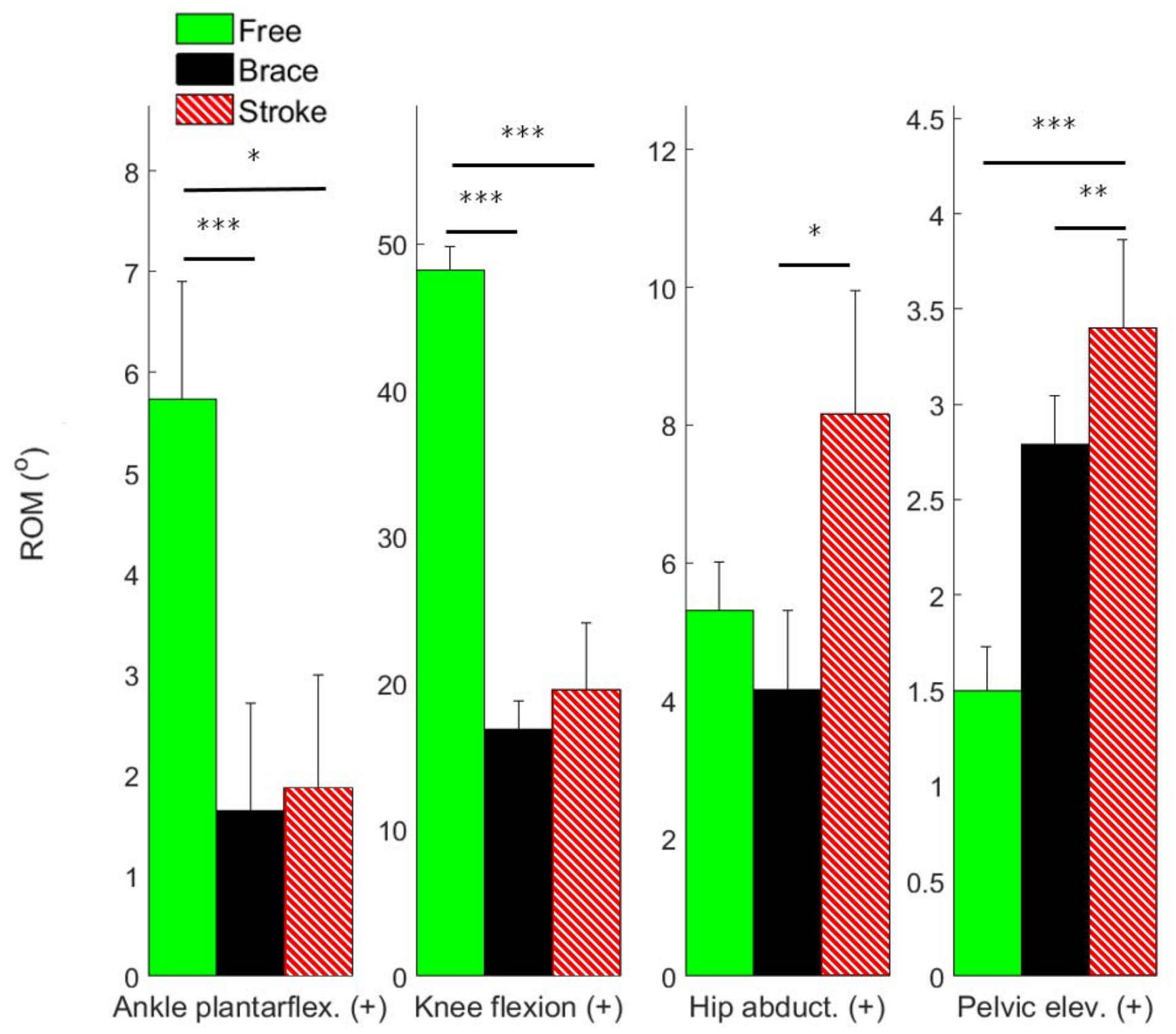

Figure S1: Similar responses in pelvic obliquity of Brace condition and post-stroke SKG but

increased hip abduction in stroke gait. The bar graphs and the error bars represent the mean

ROM values and standard errors respectively for knee flexion, ankle plantarflexion, hip abduction for constrained/paretic side and pelvic elevation in healthy individuals with Free and Brace conditions and stroke group. The level of significance between groups for given outcome measure were indicated on the lines connecting corresponding box plots ( ${ }^{*} p<0.05, * * p<$ $0.01, * * * p<0.001)$ 


\section{Tables}

\begin{tabular}{|c|c|c|c|c|}
\hline & & Free & Brace & SKG \\
\hline \multirow{5}{*}{ 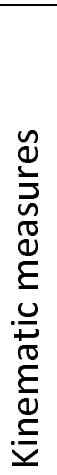 } & Knee ROM $\left({ }^{\circ}\right)$ & $48.2 \pm 5.76^{\ddagger \pm \neq,+++}$ & $16.9 \pm 6.57^{\ddagger \neq \ddagger}$ & $19.6 \pm 13.5^{++t}$ \\
\hline & Hip abd. ROM( $\left.{ }^{\circ}\right)$ & $5.31 \pm 2.46$ & $4.16 \pm 3.98^{*}$ & $8.16 \pm 5.37^{*}$ \\
\hline & Pel obl. ROM $\left.^{\circ}{ }^{\circ}\right)$ & $1.50 \pm 0.80^{\ddagger \pm, t+t}$ & $2.79 \pm 0.88^{\ddagger \ddagger}$ & $3.40 \pm 1.40^{t+t}$ \\
\hline & Ankle $\mathrm{ROM}\left({ }^{\circ}\right)$ & $5.73 \pm 4.04^{\ddagger \mp \ddagger+}$ & $1.64 \pm 3.72^{\ddagger \neq \ddagger}$ & $1.87 \pm 3.37^{+}$ \\
\hline & Peak contralateral ankle plantarflexion $\left({ }^{\circ}\right)$ & $4.62 \pm 3.40$ & $5.21 \pm 3.65$ & $5.51 \pm 5.86$ \\
\hline \multirow{6}{*}{ 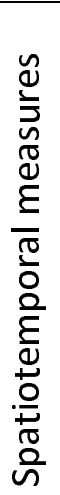 } & Max. toe height (mm) & $79.9 \pm 42.1^{\neq \neq \pm,+++}$ & $49.0 \pm 17.6^{\ddagger \neq \ddagger}$ & $35.7 \pm 11.5^{+++}$ \\
\hline & Max. toe clearance (mm) & $9.41 \pm 5.58^{\ddagger \pm \neq,+t+}$ & $10.9 \pm 7.86^{\ddagger \pm \neq, * *}$ & $21.1 \pm 9.09^{+++, * *}$ \\
\hline & Max. toe width (mm) & $29.1 \pm 9.12^{\ddagger \neq \pm,+++}$ & $39.5 \pm 11.5^{\ddagger \neq \ddagger}$ & $59.1 \pm 40.5^{++t}$ \\
\hline & Width at min. toe clearance (mm) & $-8.82 \pm 12.7^{++\dagger}$ & $-15.9 \pm 13.4^{* * *}$ & $40.1 \pm 46.8^{+++, * * *}$ \\
\hline & Swing ratio & $0.99 \pm 0.05^{\ddagger \neq \pm,+t+}$ & $1.20 \pm 0.14^{\neq \neq \pm, * * *}$ & $1.37 \pm 0.13^{t++, * * *}$ \\
\hline & Pre-swing ratio & $1.03 \pm 0.09^{t+t}$ & $1.02 \pm 0.10^{* * *}$ & $1.43 \pm 0.27^{+++, * * *}$ \\
\hline
\end{tabular}

Table 1: Summary of the joint angle ROM and spatiotemporal gait measures for healthy gait

with and without knee brace restriction and post-stroke SKG. The measures indicate the mean values and standard deviations. The level of significance between SKG and Brace condition is denoted as $* p<0.05, * * p<0.01, * * * p<0.001$; between Brace and Free conditions denoted as $\ddagger p<0.05, \ddagger \ddagger p<0.01, \ddagger \ddagger \ddagger p<0.001$; and between Free and SKG conditions denoted as $\dagger p<$ $0.05,+\dagger p<0.01,++\dagger p<0.001$ 


\section{Table S1- Demographics of healthy participants}

\begin{tabular}{cccccc} 
Subject no & Age (yrs) & G & W (lb.) & H (in) & Dominant Side \\
\hline 1 & 29 & M & 123 & 65 & $\mathrm{R}$ \\
2 & 26 & M & 163 & 69 & $\mathrm{R}$ \\
3 & 21 & $\mathrm{M}$ & 135 & 66 & $\mathrm{R}$ \\
4 & 24 & $\mathrm{M}$ & 119 & 66 & $\mathrm{R}$ \\
5 & 28 & $\mathrm{~F}$ & 164 & 71 & $\mathrm{R}$ \\
6 & 19 & $\mathrm{~F}$ & 105 & 62 & $\mathrm{R}$ \\
7 & 28 & $\mathrm{M}$ & 210 & 75 & $\mathrm{R}$ \\
8 & 27 & $\mathrm{~F}$ & 137 & 68 & $\mathrm{R}$ \\
9 & 33 & $\mathrm{M}$ & 220 & 72 & $\mathrm{~L}$ \\
10 & 22 & $\mathrm{M}$ & 145 & 69 & $\mathrm{R}$ \\
11 & 25 & $\mathrm{M}$ & 145 & 69 & $\mathrm{R}$ \\
12 & 28 & $\mathrm{M}$ & 176 & 68 & $\mathrm{R}$ \\
Mean & 25.8 & & 153 & 68.3 & \\
SD & 3.88 & & 35.1 & 3.42 & \\
\hline
\end{tabular}




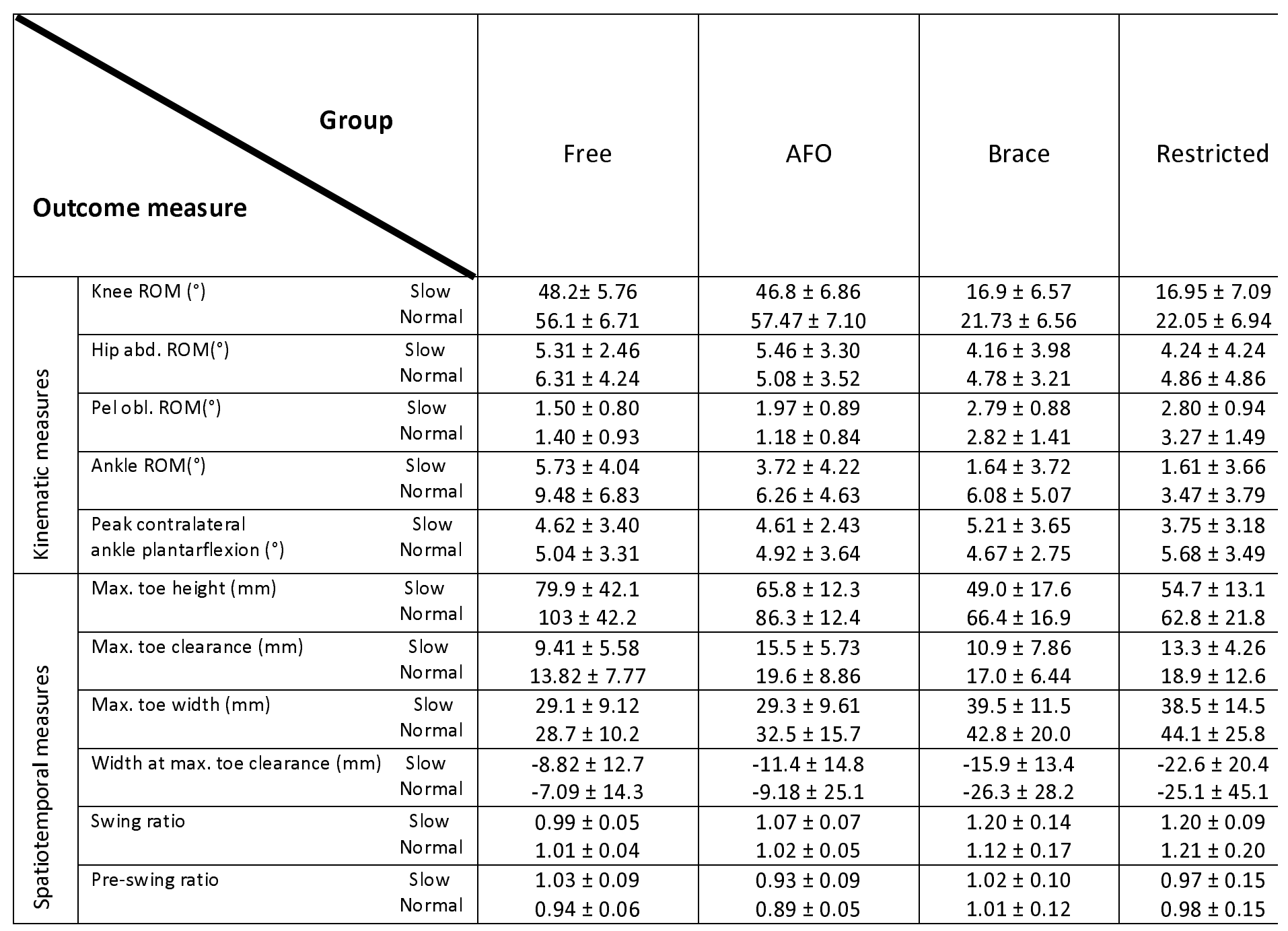

Table S2: Summary of the joint angle ROM and spatiotemporal gait measures for healthy gait

for Free, AFO, Brace and Restricted conditions with slow and normal walking speeds. The

measures indicate the mean values and standard deviations. Due to the large complexity of

markings, statistical comparisons are not noted on the table. Please see the text for the

highlighted comparisons. 